\title{
Propiedades electrónicas y estructurales del $\mathrm{Ga}_{1-\mathrm{x}} \mathrm{Cr}_{\mathrm{x}} \mathrm{As}$
}

\author{
Nasly Y. Martínez Velásquez ${ }^{1,2, *}$, Jairo Arbey Rodríguez Martínez ${ }^{1}$ \\ ${ }^{1}$ GEMA Grupo de Estudio de Materiales, Departamento de Física Universidad Nacional de Colombia, Bogotá, D.C., Colombia \\ ${ }^{2}$ CAVENDISH Grupo de Investigación Licenciatura en Matemáticas y Física, \\ Facultad de Ciencias Humanas y la Educación, Universidad de los Llanos, Villavicencio, Colombia
}

\begin{abstract}
Resumen
Mediante el uso de principios basados en la teoría del funcional de la densidad - DFT (Density Functional Theory) se calcularon las propiedades electrónicas y estructurales del compuesto $\mathrm{Ga}_{1-\mathrm{x}} \mathrm{Cr}_{\mathrm{x}} \mathrm{As}$. Empleando el método de ondas planas y la aproximación de pseudopotenciales atómicos ultra suaves se resolvieron las ecuaciones de KohnSham. Para la energía de intercambio y correlación se empleó la aproximación de gradiente generalizado, dentro de la parametrización de Perdew-Burke-Ernzerhof (PBE) tal como está implementada en el código computacional Quantum-Espresso. Al dopar GaAs con impurezas de Cr, el sistema exhibe un comportamiento tipo half-metallic. Dicho material puede ser usado en espintrónica. (C) 2018. Acad. Colomb. Cienc. Ex. Fis. Nat.
\end{abstract}

Palabras clave: Propiedades electrónicas; DFT; QE; $\mathrm{Ga}_{1-\mathrm{x}} \mathrm{Cr}_{\mathrm{x}} \mathrm{As}$

Electronic and structural properties of $\mathrm{Ga}_{1-\mathrm{x}} \mathrm{Cr}_{\mathbf{x}} \mathrm{As}$

\begin{abstract}
The structural and electronic properties of the $\mathrm{Ga}_{1-\mathrm{x}} \mathrm{Cr}_{\mathrm{x}} \mathrm{As}$ compound were calculated by employing principles based in the Density Functional Theory-DFT. In order to solve the Kohn-Sham equations, the plane wave method and the ultrasoft atomic pseudopotential approximation were used. Moreover, for the exchange and correlation energy, the generalized gradient approximation was employed, taking into account the Perdew-Burke-Ernzerhof (PBE) parametrization, as it is implemented in the computational code quantum espresso. To dope the GaAs with impurities, the system exhibits a half-metallic behavior. This material can be used in spintronics. (C) 2018. Acad. Colomb. Cienc. Ex. Fis. Nat.
\end{abstract}

Key words: Electronic properties; DFT; QE; $\mathrm{Ga}_{1-\mathrm{x}} \mathrm{Cr}_{\mathrm{x}}$ As.

\section{Introducción}

Compuestos binarios III-arsénico exhiben propiedades que los hacen atractivos para aplicaciones comerciales y tecnológicas, entre ellos se encuentra el GaAs, usado en diferentes dispositivos electrónicos y optoelectrónicos tales como diodos emisores de luz, foto detectores, láseres y circuitos integrados, entre otros, debido a que posee gab directo, (Ahmed, et al., 2007).

Los semiconductores que no poseen propiedades magnéticas, se pueden dopar con elementos magnéticos, variando así las propiedades magnéticas del semiconductor, éstos se conocen como semiconductores magnéticos diluidos (DMS Diluted Magnetic Semiconductors) (He \& HaiMing, 2008), de amplia aplicación en espintrónica. Diversos estudios realizados con el GaAs confirman que al adicionar impurezas de algunos metales de transición $3 d$, este compuesto exhibe un momento magnético considerable.

Para analizar las modificaciones de las propiedades electrónicas, estructurales y magnéticas del $\mathrm{Ga}_{1-\mathrm{x}} \mathrm{Cr}_{\mathrm{x}} \mathrm{As}$, se varió la concentración del $\mathrm{Cr}$, se trabajó con celdas $1 \times 1 \times 1$, $1 \times 1 \times 2$ y $2 \times 2 \times 2$, que corresponden a concentraciones de $\mathrm{Cr}$ de $0.25,0.125$ y 0.03125 respectivamente. Concentraciones que están de acuerdo con lo reportado por Shirai (2001), Baykov, et al. (2007) y Sandratskii \& Bruno (2003).

\section{Optimización de parámetros}

Los cálculos se realizaron con el software quantum-espresso (Giannozzi, et al., 2009), se trabajó con el formalismo de la teoría del funcional de la densidad DFT, se empleó el método de ondas planas y pseudopotenciales atómicos ultra-suaves. Se trabajó con la aproximación de Gradiente generalizado (GGA) en la parametrización de Perdew, Burke y Ernzerhof (PBE), (J. P. Perdew, et al., 1996). El GaAs se estudió en la estructura zincblenda, con una constante de red optimizada de 5.66 $\AA$ (10.85 Bohr), se empleó una malla de puntos k de 6x6x6.

Se realizó optimización de la energía de corte $E_{\text {cutoff }}$ y la malla de puntos $\mathrm{k}$ con respecto al volumen de equilibrio y a la constante de red, como se muestra en el tabla 1.

\footnotetext{
*Correspondencia:

Nasly Y. Martínez Velásquez, nmartinez@unillanos.edu.co

Recibido: 17 de agosto de 2017

Aceptado: 25 de febrero de 2018

Editor: Guillermo González Villegas
} 
Tabla 1. Energía de corte (Ecutoff), malla de puntos k Monkhorst-Pack, volumen, constante de red, módulo de volumen y energía mínima para el GaAs en su estructura zincblenda

\begin{tabular}{|c|c|c|c|c|c|}
\hline \multirow[t]{2}{*}{ Ecutoff } & \multirow[t]{2}{*}{ Parámetros } & \multicolumn{4}{|c|}{ Malla puntos $k$} \\
\hline & & $3 \times 3 \times 3$ & $4 \times 4 \times 4$ & $6 \times 6 \times 6$ & $10 \times 10 \times 10$ \\
\hline \multirow[t]{5}{*}{$25 \mathrm{Ry}$} & $\mathrm{a}_{\min }(\mathrm{Bohr})$ & 10,851 & 10,849 & 10,849 & 10,850 \\
\hline & $\mathrm{v}_{\min }\left(\mathrm{bohr}_{3}\right)$ & 319,41 & 319,25 & 319,25 & 319,34 \\
\hline & Bo (Mbar) & 0,62 & 0,62 & 0,60 & 0,61 \\
\hline & Bo' & 3,96 & 4,27 & 4,70 & 4,48 \\
\hline & $\mathrm{E}_{\min }(\mathrm{eV})$ & $-2910,71$ & $-2810,73$ & $-2910,73$ & $-2910,73$ \\
\hline \multirow[t]{5}{*}{30 Ry } & $\mathrm{a}_{\min }(\mathrm{Bohr})$ & 10,850 & 10,849 & 10,850 & 10,850 \\
\hline & $\mathrm{v}_{\min }\left(\mathrm{bohr}_{3}\right)$ & 319,40 & 319,26 & 319,36 & 319,39 \\
\hline & Bo (Mbar) & 0,61 & 0,61 & 0,60 & 0,60 \\
\hline & Bo & 4,38 & 4,68 & 4,64 & 4,63 \\
\hline & $\mathrm{E}_{\min }(\mathrm{eV})$ & $-2910,79$ & $-2810,81$ & $-2910,81$ & $-2910,81$ \\
\hline \multirow[t]{5}{*}{35 Ry } & $\mathrm{a}_{\min }(\mathrm{Bohr})$ & 10,851 & 10,849 & 10,850 & 10,851 \\
\hline & $\mathrm{v}_{\min }\left(\mathrm{bohr}_{3}\right)$ & 319,33 & 319,31 & 319,39 & 319,40 \\
\hline & Bo (Mbar) & 0,61 & 0,61 & 0,61 & 0,60 \\
\hline & Bo’ & 4,60 & 4,60 & 4,60 & 4,60 \\
\hline & $\mathrm{E}_{\min }(\mathrm{eV})$ & $-2910,80$ & $-2810,81$ & $-2910,82$ & $-2910,82$ \\
\hline \multirow[t]{5}{*}{40 Ry } & $\mathrm{a}_{\min }(\mathrm{Bohr})$ & 10,849 & 10,849 & 10,850 & 10,850 \\
\hline & $\mathrm{v}_{\min }\left(\mathrm{bohr}_{3}\right)$ & 319,31 & 319,30 & 319,37 & 319,39 \\
\hline & Bo (Mbar) & 0,61 & 0,61 & 0,61 & 0,60 \\
\hline & Bo' & 4,61 & 4,61 & 4,60 & 4,61 \\
\hline & $\mathrm{E}_{\min }(\mathrm{eV})$ & $-2910,80$ & $-2810,82$ & $-2910,82$ & $-2910,82$ \\
\hline
\end{tabular}

\section{Resultados y discusiones}

Teniendo en cuenta los resultados que se presentan en el cuadro 1, se grafica EvsV para cada una de las energías de corte (figura 1). El valor de la constante de red que hace mínima la energía se encuentra a partir de una malla de puntos k de 6x6x6, para valores de $E_{\text {cutoff }}$ de $30 \mathrm{Ry}, 35$ Ry y $40 \mathrm{Ry}$, de igual manera para las energías de corte mencionadas, con una malla de puntos k de 10x10x10 el valor de la constante de red es similar al encontrado con la malla de $6 \times 6 \times 6$. Teniendo en cuenta lo mencionado y el coste computacional al correr los cálculos con los parámetros descritos, se selecciona la $E_{\text {cutoff }}$ de 30 Ry con una malla de puntos $\mathrm{k}$ de 6x6x6, para el valor de a que minimiza la energía correspondiente al $\mathrm{a}_{0}(10.85 \mathrm{bohr})$.

En el tabla 2, se compara el valor de la constante de red y el módulo de volumen calculado, para el GaAs en la estructura zincblenda, con otros datos reportados en la literatura. Se relacionan los porcentajes de error para cada uno de los parámetros escogidos.

Los valores reportados por Ahmed, et al., (2007) son de carácter teórico, calculados mediante el método FP-LDA y FP-GGA por sus siglas en inglés Full-potential linear augmented plane wave (FP-LAPW) con la aproximación de densidad local (LDA - Local density aproximation) y aproximación de gradiente generalizado (GGA -generalized gradiente approximation), respectivamente, cálculos realizados mediante código WIEN2K; los datos reportados por Hellwege, et al., (1982) y Fillipi, et al., (1994) son de carácter experimental.
La diferencia en los valores obtenidos se debe a los métodos empleados para hallar dicho parámetro.

\section{Propiedades electrónicas y estructurales}

Las gráficas de bandas de energía que se presentan, se hicieron para el camino $\Gamma, \mathrm{M}, \mathrm{X}, \Gamma, \mathrm{R}, \mathrm{X}, \mathrm{M}, \mathrm{R}$; camino de alta simetría de la Primera Zona de Brillouin, lo enunciado se trabajó para la celda zincblenda y la supercelda $2 \times 2 \times 2$. Para la supercelda $1 \times 1 \times 2$, se tuvo en cuenta el camino de alta simetría $\Gamma, \mathrm{M}, \mathrm{X}, \Gamma, \mathrm{A}, \mathrm{M}, \mathrm{X}, \mathrm{A}$, en la PZB.

A continuación se presentan los resultados para el GaAs en su estructura zincblenda.

De acuerdo con la figura 2 el GaAs posee gap directo en $\Gamma$, con un valor de $0.25 \mathrm{eV}$, dicho valor permite ver que el compuesto es un semiconductor; éste resultado concuerda con otros datos teóricos reportados por Ahmed, et al., (2007); Grivickas, et al., (2009); Ihm \& Joannopoulus (1981); Sze \& Ng (2007); Wang \& Klein (1981). Resultados similares se encuentran para las superceldas $1 \times 1 \times 2$ y $2 \times 2 \times 2$.

$\mathrm{Al}$ sustituir un átomo de $\mathrm{Ga}$ por uno de $\mathrm{Cr}$ en la red, se encuentra que las bandas de energía difieren de la estructura pura. Se encuentra que para una orientación de espín presenta un comportamiento conductor, y para la otra, un comportamiento aislante R.A.Groot, et al. (1983); Sandratskii \& Bruno (2003), (figura 3), es decir el sistema exhibe un comportamiento half-metallic.

En la figura 3(a) se observa un traslapamiento de bandas de valencia y conducción, hecho que evidencia un carácter metálico del $\mathrm{Ga}_{1-\mathrm{x}} \mathrm{Cr}_{\mathrm{x}} \mathrm{As}(x=0 ; 25)$. Cerca al nivel de Fermi 


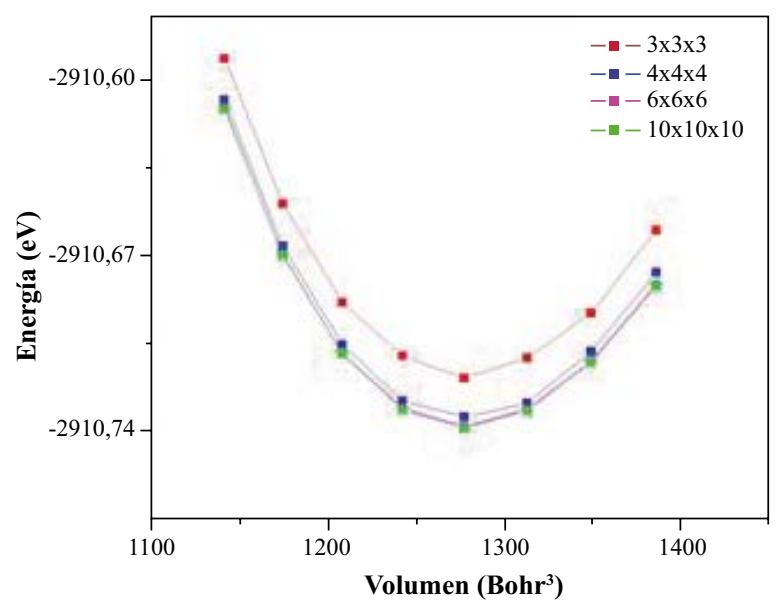

(a)

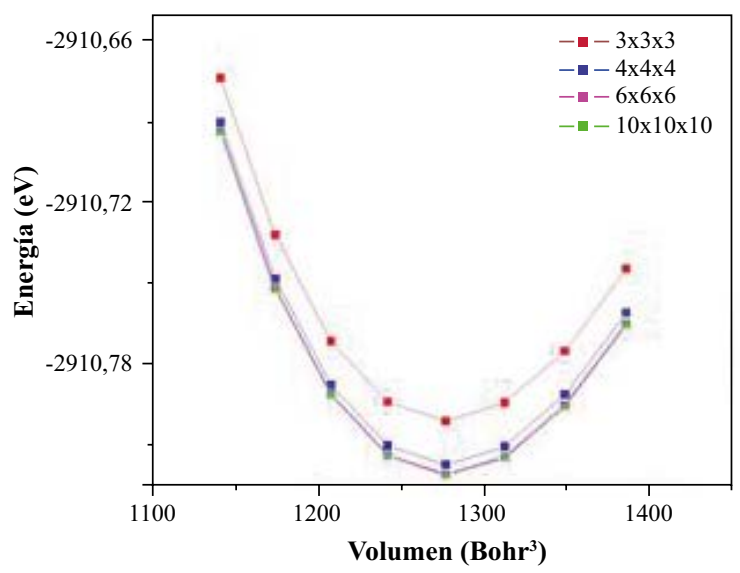

(c)

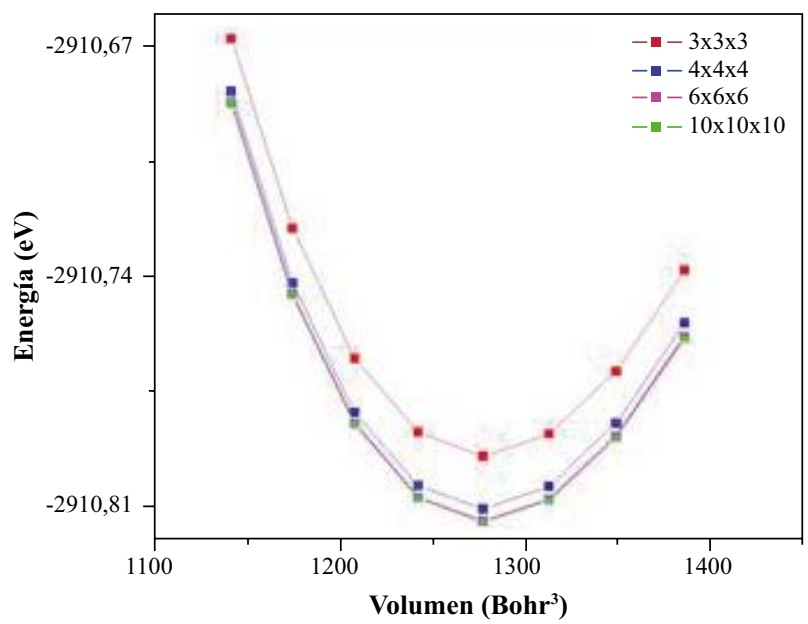

(b)

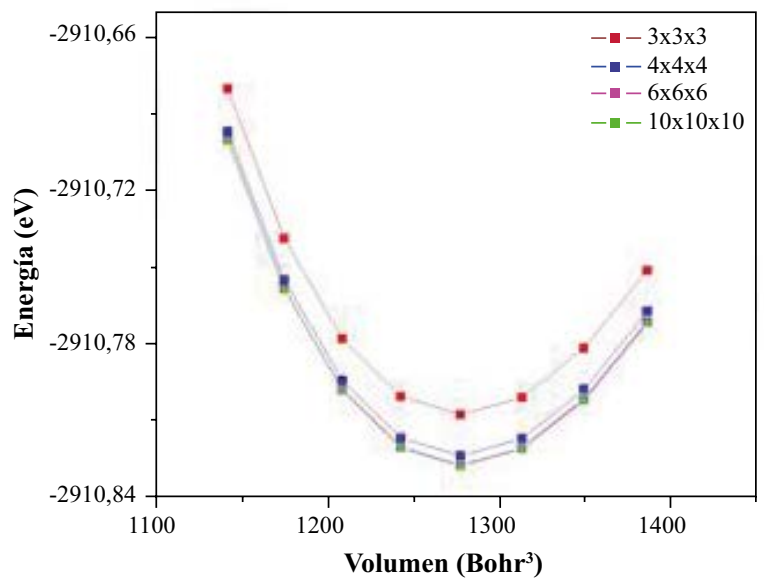

(d)

Figura 1. Energía Vs Volumen para el GaAs en su estructura zincblenda. Para una energía de corte de (a) 25 Ry, (b) 30 Ry, (c) 35 Ry y (d) 40 Ry.

Tabla 2. Constante de red, módulo de volumen, método de cálculo, porcentaje de error para el GaAs

\begin{tabular}{|c|c|c|c|c|}
\hline $\mathbf{a}_{0}(\mathrm{~A})$ & $\mathrm{B}_{0}$ (GPa) & Método & $\% \mathbf{a}_{0}(\mathrm{~A})$ & $\% B_{0}(G P a)$ \\
\hline 5,608 Ahmed, et al. (2007) & 75,2 Ahmed, et al. (2007) & FP-LDA & $0,94 \%$ & $-18,94 \%$ \\
\hline 5,748 Ahmed, et al. (2007) & 60,8 Ahmed, et al. (2007) & FP-GGA & $-1,51 \%$ & $0,24 \%$ \\
\hline 5,654 Hellwege, et al. (1982) & 77,0 Hellwege, et al. (1982) & Experimento & $0,12 \%$ & $-20,84 \%$ \\
\hline 5,653 Fillipi, et al. (1994) & 76,0 Fillipi, et al. (1994) & Experimento & $0,14 \%$ & $-19,80 \%$ \\
\hline $5,66^{*}$ & 60,95 & Pseudopotenciales & & \\
\hline
\end{tabular}

* Presente trabajo

y hasta $-4 \mathrm{eV}$, la contribución de orbitales corresponde a orbitales $4 \mathrm{p}$ del Ga, $4 \mathrm{p}$ del As y $3 \mathrm{~d}$ del Cr.

En la figura 3(b) se observan tres regiones por debajo del nivel de Fermi. La región más cerca al nivel de Fermi, entre $-0.7 \mathrm{eV}$ y $-4 \mathrm{eV}$, tiene la mayor contribución en electrones $4 \mathrm{p}$ del As y $4 \mathrm{p}$ del Ga, la contribución de electrones 3d no es mayoritaria como en el caso de las bandas con spin up, se observa la presencia de un gap de energía.
Al analizar las gráficas de bandas de energía para el $\mathrm{Ga}_{1-\mathrm{x}} \mathrm{Cr}_{\mathrm{x}} \mathrm{As}$ (graficas 3 (a) y 4(a)), sustituyendo un átomo de Ga por uno de $\mathrm{Cr}$ en las estructuras 1x1x1 y 1x1x2 respectivamente, se confirma el comportamiento "halfmetallic" del sistema.

En la figura 3(b) se observan tres regiones por debajo del nivel de Fermi. La región mas cerca al nivel de Fermi, entre $-0.7 \mathrm{eV}$ y $-4 \mathrm{eV}$, tiene la mayor contribución en electrones 


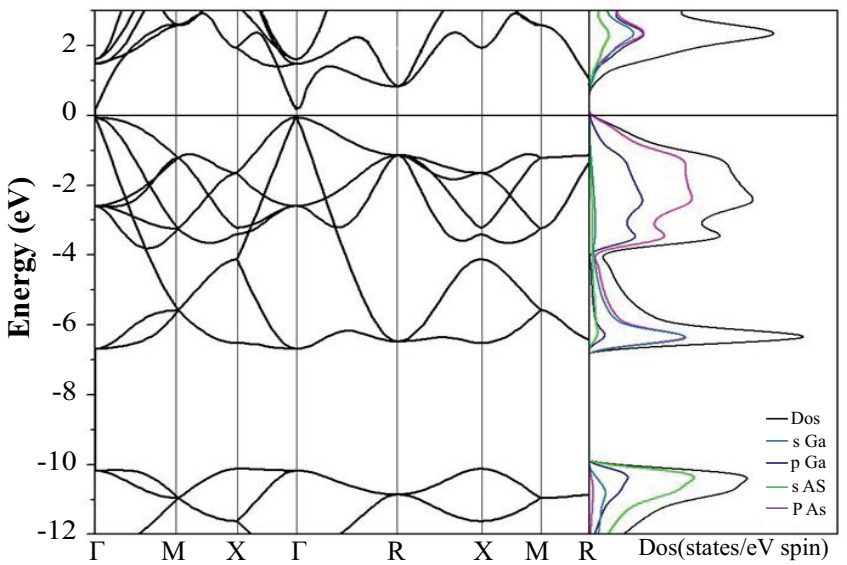

Figura 2. Bandas de energía para el GaAs $1 \times 1 \times 1$

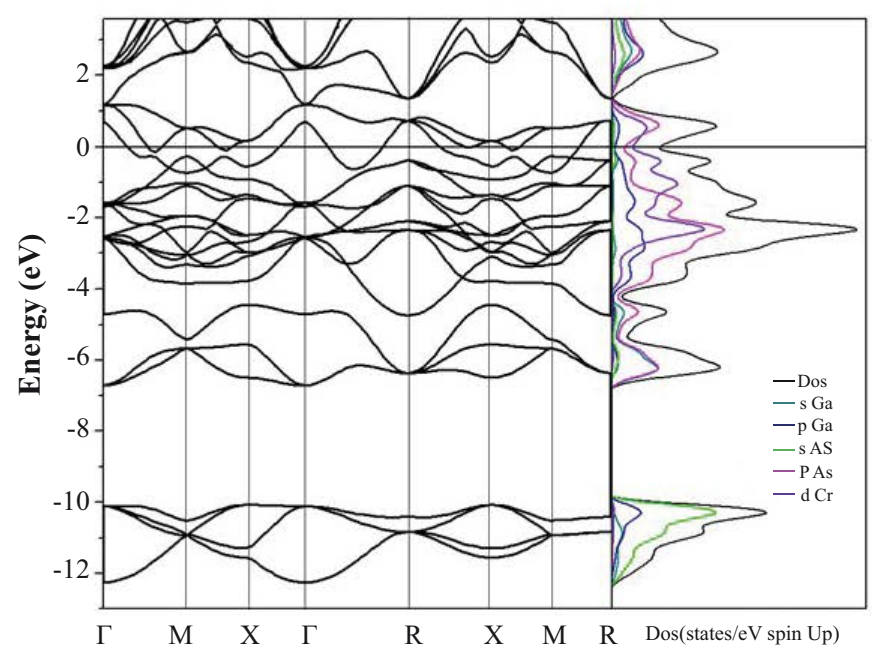

(a)
$4 \mathrm{p}$ del As y $4 \mathrm{p}$ del Ga, la contribución de electrones $3 \mathrm{~d}$ no es mayoritaria como en el caso de las bandas con spin up, se observa la presencia de un gap de energía

Al analizar las gráficas de bandas de energía para el $\mathrm{Ga}_{1-\mathrm{x}} \mathrm{Cr}_{\mathrm{x}} \mathrm{As}$ ((gráficas 3 (a) y 4(a)), sustituyendo un átomo de $\mathrm{Ga}$ por uno de $\mathrm{Cr}$ en las estructuras $1 \mathrm{x} 1 \mathrm{x} 1$ y $1 \times 1 \times 2$ respectivamente, se confirma el comportamiento "halfmetallic" del sistema.

Al hacer la sustitución de un átomo de $\mathrm{Ga}$ por uno de $\mathrm{Cr}$ en la supercelda $2 \times 2 \times 2$, la concentración del dopante es de 0.03125 . Al comparar las figuras 3,4 y 5, se encuentra que la contribución de orbitales es similar para las estructuras 1x1x1, 1x1x2 y 2x2x2 como se ha descrito en párrafos anteriores, al sustituir un átomo de galio por uno de cromo, el compuesto presenta magnetización.

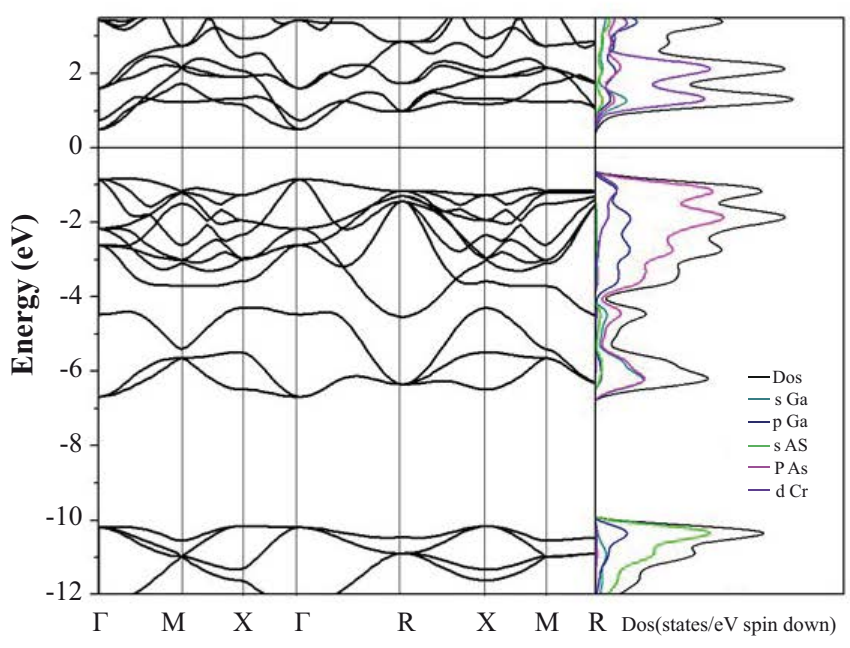

(b)

Figura 3. Bandas de energía para el GaAs $1 \times 1 \times 1$ spin up (a) y spin down (b), al sustituir un átomo de Ga por uno de Cr.

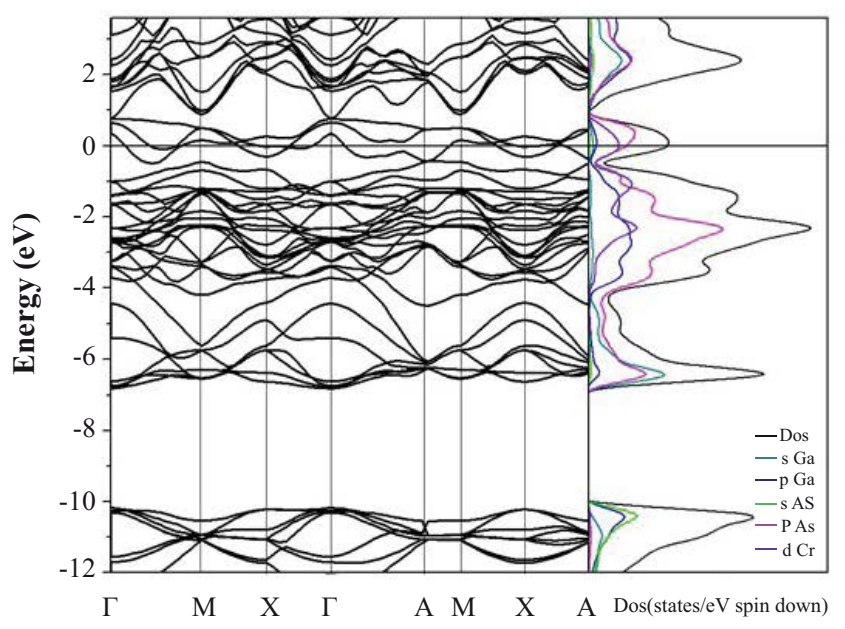

(a)

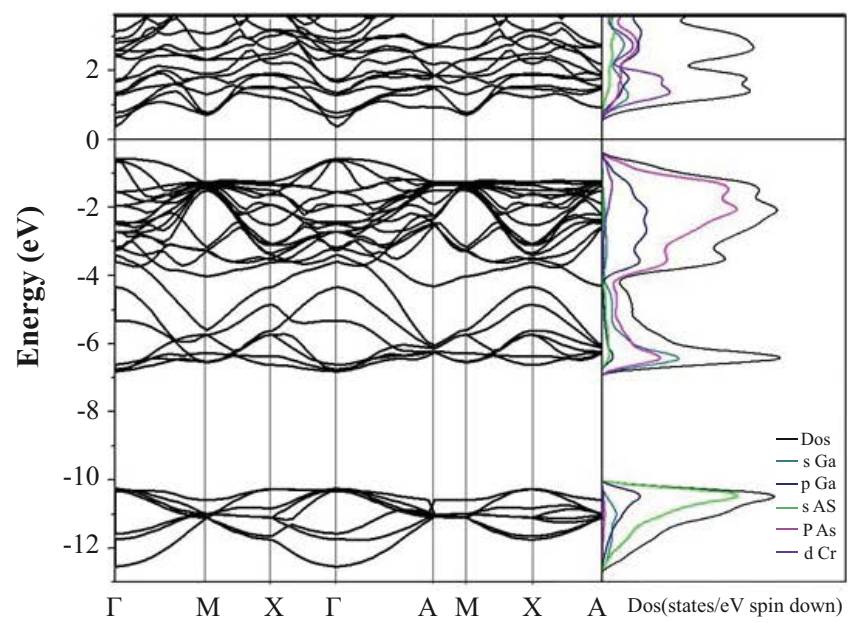

(b)

Figura 4. Bandas de energía para el GaAs 1x1x2 spin up (a) y spin down (b), reemplazando un atomo de Ga por uno de Cr. 


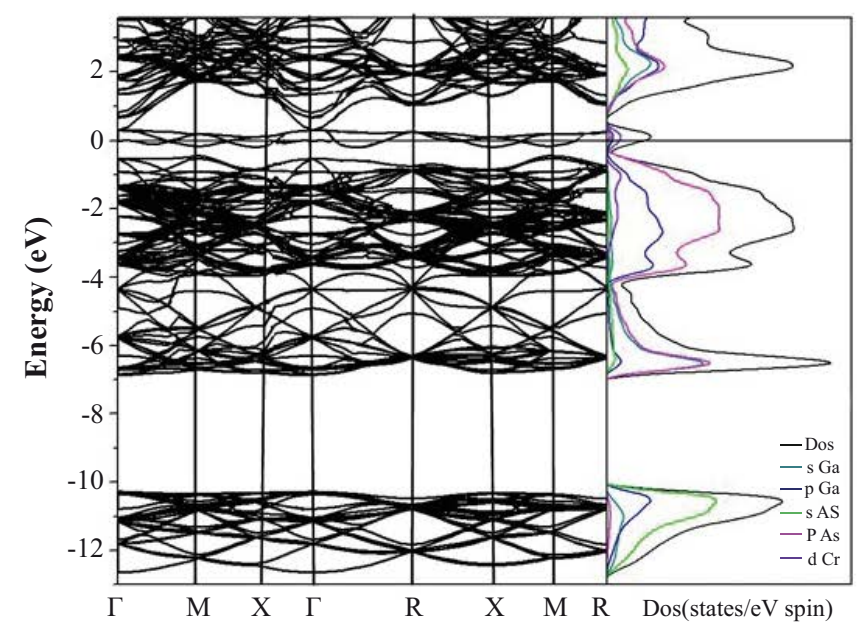

(a)

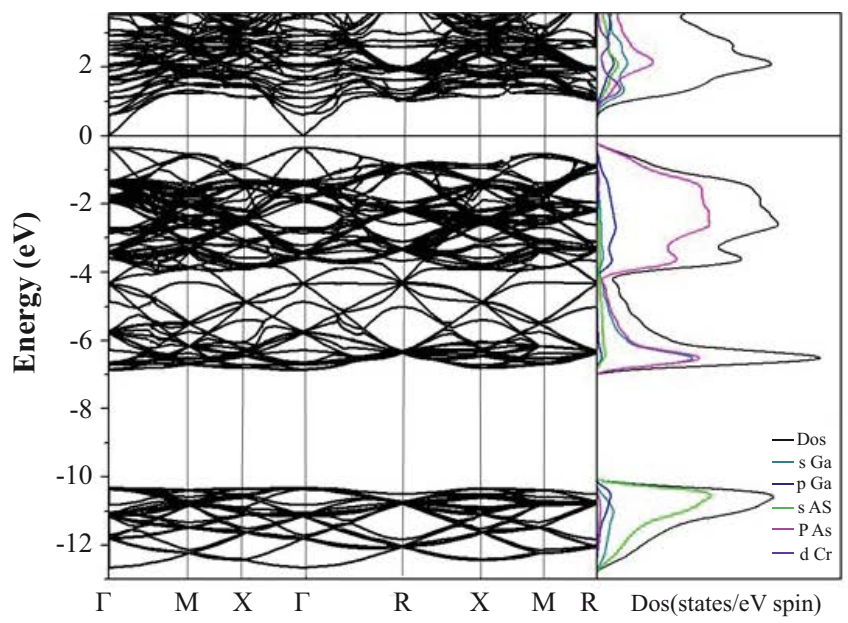

(b)

Figura 5. Bandas de energía para el GaAs $2 \times 2 \times 2$ spin up (a), spin down, al hacer una sustitución de un átomo de Ga por uno de Cr.

En el tabla 3 se presenta la variación de la constante de red en función de la concentración, para las estructuras estudiadas.

Al hacer la sustitución de un átomo de $\mathrm{Ga}$ por uno de $\mathrm{Cr}$, se encuentra que la constante de red no varía significativamente (analizando el caso en cada estructura), esto se debe a que la distribución de carga es similar para el átomo de $\mathrm{Ga}$ y el de $\mathrm{Cr}$.

En el tabla 4 se presenta el momento magnético en $\mathrm{Ga}_{1-\mathrm{x}} \mathrm{Cr}_{\mathrm{x}} \mathrm{As}$, para las tres concentraciones trabajadas $\mathrm{x}=0.25$, $\mathrm{x}=0.125 \mathrm{y} \mathrm{x}=0.03125$. Se muestra el momento magnético de la impureza $3 \mathrm{~d}$, quien induce momento en los átomos vecinos de As. Igualmente se presenta el momento magnético total de la celda.

El momento magnético total en los casos presentados al hacer la sustitución de un átomo de $\mathrm{Ga}$ por uno de $\mathrm{Cr}$ en las estructuras consideradas es de $3 \mu \mathrm{B}$, independiente

Tabla 3. Constante de red para el GaAs con una concentración de $\mathrm{Cr}$ de $0.25,0.125$ y 0.03125 , correspondientes a la estructura zincblenda, y a las superceldas $1 \times 1 \times 2$ y $2 \times 2 \times 2$ respectivamente.

\begin{tabular}{lcc}
\hline Constante de Red & GaAs & GaAs Ga $\mathbf{C r}_{\mathbf{C r}}$ \\
\hline a (Bohr) $1 \times 1 \times 1$ & 10,99 & 10,97 \\
a (Bohr) $1 \times 1 \times 2$ & 10,98 & 10,99 \\
a (Bohr) $2 \times 2 \times 2$ & 10,98 & 11,00 \\
\hline
\end{tabular}

Tabla 4. Momento magnético en $\mathrm{Ga}_{1-\mathrm{x}} \mathrm{Cr}_{\mathrm{x}} \mathrm{As}$. Las unidades del momento magnético son $\mathrm{B}$.

\begin{tabular}{llccc}
\hline & & $\mathbf{X}=\mathbf{0 , 2 5}$ & $\mathbf{X = 0 , 1 2 5}$ & $\mathbf{X = 0 , 0 3 1 2 5}$ \\
\hline \multirow{4}{*}{$(\mathrm{GaCr}) \mathrm{As} s$} & $\mathrm{Cr}$ & 3,45 & 3,46 & 3,55 \\
& AS & $-0,17$ & $-0,15$ & $-0,15$ \\
& Celda & 3,00 & 3,00 & 3,00 \\
\hline
\end{tabular}

de la concentración del dopante, esto está de acuerdo con lo reportado por Sandratskii (Sandratskii \& Bruno, 2003), y por De-ming Ma (De-min Ma \& Shi, 2016); la magnetización se presenta por la hibridación de los orbitales As-4p y Cr-3d.

\section{Conclusiones}

El GaAs en su estructura zincblenda, posee gap directo. Este hecho lo convierte en un material atractivo para su uso en celdas solares, heteroestructuras para emisores de luz, entre otros. Cerca al nivel de Fermi para este material se encuentra que la contribución electrónica corresponde a electrones $4 p$ del As y $4 \mathrm{p}$ del $\mathrm{Ga}$, y estando aun en el core, entre $-4 \mathrm{eV}$ y $-7 \mathrm{eV}$ la contribución corresponde a electrones $4 p$ del As y 4 s del Ga.

Dicho material no presenta magnetización. Al hacer la sustitución de un átomo de Ga por uno de $\mathrm{Cr}$ para diferentes concentraciones de dopante, el material presenta un comportamiento "half-metallic", dicho material puede ser usado como un semiconductor magnético diluido, como inyector de espín, también es un material que se puede usar en aplicaciones en espintrónica.

La magnetización se presenta por la hibridacion de orbitales As-4p y Cr-3d.

\section{Contribución de los autores}

NYMV, redactó los resultados del trabajo producto de la tesis de maestría, ajustó el texto de acuerdo con las recomendaciones del director y actualizó las referencias. JARM, llevó a cabo el análisis del compuesto $\mathrm{Ga}_{1-\mathrm{x}} \mathrm{Cr}_{\mathrm{x}} \mathrm{As}$, sus propiedades electrónicas, estructurales y magnéticas, redactó el artículo, revisó el texto e hizo las correcciones y ajustes.

\section{Conflicto de intereses}

Los autores declaran no tener conflicto de intereses con respecto al contenido de este artículo original. 


\section{Referencias}

Ahmed, R., Hashemifar, S. J., Akbarzadeh, H., Ahmed, M. \& e Aleem, F. (2007). Ab initio study of structural and electronic properties of iii-arsenide binary compounds. Elsevier ScienceDircet.Computational Materials Science 39, 580586.

Baykov, V. I., Korzhavyi, P. A., Smirnova, E. A., Abrikosov, I. A. \& Johansson, B. (2007). Magnetic properties of 3d impurities in gaas. Journal of Magnetism and Magnetic Materials 310 (3): 2120-2122.

De-min Ma, V. W. E.-l. L., Yong-yong Chai \& Shi, W. (2016). Electornic structure, magnetic and optical properties of crdoped GaAs using hybrid density functional. omputational Materials Science 113: 75-79.

Fillipi, B., Singh, D. \& Umrigar, C. (1994). Phys. Rev. B 50: 14947.

Giannozzi, P., Baroni, S., Bonini, N., Calandra, M., Car, R., Cavazzoni, C., Ceresoli, D., Chiarotti, G. L., Cococcioni, M., Dabo, I., Corso, A. D., de Gironcoli, S., Fabris, S., Fratesi, G., Gebauer, R., Gerstmann, U., Gougoussis, C., Kokalj, A., Lazzeri, M., Martin-Samos, L., Marzari, N., en Francesco Mauri, Mazzarello, R., Paolini, S., Pasquarello, A., Paulatto, L., Sbraccia, C., Scandolo, S., Sclauzero, G., Seitsonen, A. P., Smogunov, A., Umari, P. \& Wentzcovitch, R. M. (2009). Quantum espresso: a modular and open-source software project for quantum simulations of materials. Journal of Physics: Condensed Matter 39, 395502, 19pp.
Grivickas, P., McCluskey, M. D. \& Gupta, Y. M. (2009). Transformation of GaAs into an indirect 1-band-gap semiconductor under uniaxial strain. Physical Review B 80.

He, L. \& HaiMing, D. (2008). Local electronic structure and magnetic properties of $3 \mathrm{~d}$ transition metal doped GaAs. Sci China Ser G-Phys Mech Astron. 51 (5): 470-480.

Hellwege, K., Madelung, O. \& Börnstein, L. (1982). New Series Group III, vol 17a.

Ihm, J. \& Joannopoulus, J. D. (1981). Ground-state properties of gaas and alas. Physical Review B. 24 (8).

J.P.Perdew, K.Burke \& Emzerhof, M. (1996). Phys Rev. Lett 77: 3856.

R.A.Groot, F.M.Mueller, Engen, P. \& Buschow, K. (1983). New class of materials: Half-metallic ferromagnets. Physical Review Letters 50 (25).

Sandratskii, L. M. \& Bruno, P. (2003). Electronic structure, exchange interactions, and curie temperature in diluted III-V magnetic semiconductors: (GaCr)As, (GaMn)As, (GaFe)As. Physical Review B. 67: 214402.

Shirai, M. (2001). Electronic and magnetic properties of $3 \mathrm{~d}$ transition-metal-doped gaas. Physica E. 10: 143-147.

Sze, S. M. \& Ng, K. K. (2007). Physics of semiconductor devices. Third Edition. John Wiley and Sons.

Wang, C. S. \& Klein, B. M. (1981). First principles electronic structure of $\mathrm{Si}, \mathrm{Ge}, \mathrm{GaP}, \mathrm{GaAs}, \mathrm{ZnS}$ and $\mathrm{ZnSe}$. II optical porperties. Phys. Rev. B. 24 (6): 3417-3429. 